\title{
Averages of ratios compared to \\ ratios of averages: \\ mathematical results
}

by

L. Egghe

Universiteit Hasselt (UHasselt), Campus Diepenbeek, Agoralaan, B-3590 Diepenbeek, Belgium $^{1}$

and

Universiteit Antwerpen (UA), IBW, Stadscampus, Venusstraat 35, B-2000 Antwerpen, Belgium

leo.egghe@uhasselt.be

\section{ABSTRACT}

The recently awakened discussion on the usability of averages of ratios ( $A o R$ ) compared to ratios of averages ( $R o A$ ) has lead to the mathematical results in this paper. Based on the empirical results in Larivière and Gingras (Journal of Informetrics, 2011 to appear) we prove, under reasonable conditions, the following relations between $A o R$ and $R o A$ for a set of points:

(i) The regression line of $R o A$ in function of $A o R$ is the first bissectrix

(ii) $(A o R-R o A) / A o R$ in function of the number $N$ of papers is a cloud of points comprised between a multiple of $1 / \sqrt{N}$ and $-1 / \sqrt{N}$

(iii) $(A o R-R o A) / A o R$ versus $R o A$ has a decreasing regression line.

\footnotetext{
${ }^{1}$ Permanent address

Key words and phrases: average of ratios, ratio of averages, global impact factor, average impact factor, crown indicator
} 


\section{Introduction}

The basis of this paper is mathematical: let us have $N$ numbers $x_{1}, x_{2}, \ldots, x_{N}$ and $N$ numbers $y_{1}, y_{2}, \ldots, y_{N}(N=1,2,3, \ldots)$. All these numbers are supposed to be strictly positive numbers (but not necessarily natural numbers). Their average of ratios $(A o R)$ is defined as in formula (1)

$$
A o R=\frac{1}{N} \sum_{i=1}^{N} \frac{y_{i}}{x_{i}}
$$

, the average of the $\frac{y_{i}}{x_{i}}$-values.

The terminology speaks for itself. Their ratio of averages $(R o A)$ is defined as in formula (2)

$$
\operatorname{RoA}=\frac{\sum_{i=1}^{N} y_{i}}{\sum_{i=1}^{N} x_{i}}
$$

The name of $R o A$ comes from the fact that

$$
\operatorname{RoA}=\frac{\frac{1}{N} \sum_{i=1}^{N} y_{i}}{\frac{1}{N} \sum_{i=1}^{N} x_{i}}=\frac{\bar{y}}{\bar{x}}
$$

, the division of the average of the $y_{i}$-values and the average of the $x_{i}$-values $(i=1, \ldots, N)$.

The difference between $A o R$ and $R o A$ has already been studied in Egghe and Rousseau (1996a,b) in the connection of averages of impact factors (IFs) of $N$ journals. There we have $N$ journals and, for each $i=1, \ldots, N, x_{i}=P_{i}$ is the number of articles in journal $i$ and $y_{i}=C_{i}$ is the number of citations to these articles (one must specify the publication period and citation period but for the mathematical theory on (1) and (2) in this connection, this is not important). In this interpretation, (1) becomes

$$
A I F=\frac{1}{N} \sum_{i=1}^{N} \frac{C_{i}}{P_{i}}
$$

, the average impact factor of these $N$ journals, and (2) becomes 


$$
G I F=\frac{\sum_{i=1}^{N} C_{i}}{\sum_{i=1}^{N} P_{i}}
$$

, the global impact factor of these $N$ journals. The main result in Egghe and Rousseau (1996a) is the characterization of $A I F>G I F, A I F=G I F, A I F<G I F$ in terms of the slope of the regression line of the cloud of points $\left(P_{i}, \frac{C_{i}}{P_{i}}\right)$ for $i=1, \ldots, N$. This basic result is, obviously, also valid in the general setting (1) and (2) since (4) and (5) only differ from (1) and (2) in notation. We will repeat this result in the next section on which our basic assumption on a cloud of points $\left(A o R_{j}, R o A_{j}\right)$ for $j=1, \ldots M$ will be based. This basic assumption is then capable of explaining the three graphs in Larivière and Gingras (2011) (more details to follow). Also in Egghe and Rousseau (1996b) we noticed that, if we replace in (4) and (5) the arithmetic averages (see also (1) and (3)) by geometric averages, the formulae (4) and (5) are identical, which is a clear advantage of geometric averages on rankings based on (4) or (5) (since for geometric averages the ranks are the same) (see also the conclusions section).

Much more recent is the debate around the difference between (1) and (2), interpreted as "crown indicator" (for (2)) and "new crown indicator" (for (1)). The classical crown indicator, used by the CWTS group for years, is (2) where $N$ is the number of papers (e.g. of an author or institute), $y_{i}=C_{i}$ is the number of citations to the $i^{\text {th }}$ paper and where $x_{i}=f_{i}$ is a field average in the field of paper $i$ (average number of citations per paper in this field). Here we have the notation

$$
C I=\frac{\sum_{i=1}^{N} C_{i}}{\sum_{i=1}^{N} f_{i}}
$$

As in (5), CI takes into account the actual number of citations to each paper and hence can be considered as a "weighted" average in comparison with (1) or (4). The debate (in which we do not want to interfere) started in Opthof and Leydesdorff (2010) where the use of (1) is advocated, instead of (6). In this notation (1) becomes ("new crown indicator")

$$
C I^{*}=\frac{1}{N} \sum_{i=1}^{N} \frac{C_{i}}{f_{i}}
$$


, see also Waltman et al. (2011a) and several "Letters to the Editor" in Journal of Informetrics (issues 4(3), 4(4) and 5(1)). As said, we do not wish to go into this debate but it was the basis for the paper Larivière and Gingras (2011) for which we will give some mathematical explanations for their graphs (3 types) involving $C I$ and $C I^{*}$ or, in general terminology: $R o A$ and $A o R)$.

Another interpretation of (1) and (2) is given in Rousseau and Leydesdorff (2011) where $N$ is the number of years, $y_{i}=C_{i}$ is the number of citations to $i$ years ago of a fixed journal $J$ and $x_{i}=P_{i}$ is the number of publications in journal $J, i$ years ago $(i=1, \ldots, N)$ (and similar for other impact factors).

Also in Egghe and Rousseau (2002/2003), different interpretations (examples) of AoR versus $R o A$ are given. There, also relative variants of these indicators are given.

In view of the different interpretations above, we will, in the sequel, use the general notation (1) and (2) and we will explain three types of graphs that occur in Larivière and Gingras (2011). In the next section we will repeat the main result on the relation between AIF and GIF (in the general notation $A o R$ and $R o A$ ). On this result we will base ourselves to make a simple assumption ("axiom") on a cloud of points $\left(A o R_{j}, \operatorname{RoA}_{j}\right), j=1, \ldots, M$. This is then used to explain three types of graphs in Larivière and Gingras (2011). In the third section we will prove that the regression line of $R o A$ in function of $A o R$ is the first bissectrix. In the fourth section we show that the relation $(A o R-R o A) / A o R$ in function of the number $N$ (of papers in the crown indicator terminology) is a cloud of points comprised between a multiple of $1 / \sqrt{N}$ and $-1 / \sqrt{N}$. In the fifth section we prove that $(A o R-R o A) / A o R$ versus $R o A$ has a decreasing regression line.

The paper ends with a conclusions and comments section. 


\section{Basic assumption on a cloud of points $\left(\operatorname{AoR}_{\mathrm{j}}, \operatorname{RoA}_{\mathrm{i}}\right)_{2}$ $j=1, \ldots, M$ based on the main result in Egghe and $\underline{\text { Rousseau (1996a) }}$}

In Egghe and Rousseau (1996a) we used the notation of formulae (4) and (5) but in this paper we will use the general notation as in formulae (1) and (2). In this notation, we proved in Egghe and Rousseau (1996a) the following basic result.

\section{Theorem 1 (Egghe and Rousseau (1996a)):}

If $z=a+b x$ denotes the regression line of the cloud of points $\left(x_{i}, \frac{y_{i}}{x_{i}}=z_{i}\right)$ for $i=1, \ldots, N$, then the following assertions are equivalent:

(i) $\quad \operatorname{RoA}>A o R$ if and only if $b>0$

(ii) $R o A=A o R$ if and only if $b=0$

(iii) $R o A<A o R$ if and only if $b<0$

Let us have $M$ cases $\left(x_{1}^{(j)}, x_{2}^{(j)}, \ldots, x_{N_{j}}^{(j)}\right)$ and $\left(y_{1}^{(j)}, y_{2}^{(j)}, \ldots, y_{N_{j}}^{(j)}\right), j=1, \ldots, M$. Let us denote

$$
\alpha_{j}=A o R_{j}=\frac{1}{N_{j}} \sum_{i=1}^{N_{j}} \frac{y_{i}^{(j)}}{x_{i}^{(j)}}
$$

and

$$
\beta_{j}=\operatorname{RoA}_{j}=\frac{\sum_{i=1}^{N_{j}} y_{i}^{(j)}}{\sum_{i=1}^{N_{j}} x_{i}^{(j)}}
$$

for $j=1, \ldots, M$, representing $M$ cases where (1) and (2) can be calculated (as in (7) and (8)). Based on Theorem $1, \alpha_{j} \leq \beta_{j}$ can occur as well as $\alpha_{j} \geq \beta_{j}(j=1, \ldots, M)$. We therefore make the following basic assumption. 


\section{Assumption:}

For all points $\left(\alpha_{j}, \beta_{j}\right)$ where $\alpha_{j}$ is fixed, the average of the $\beta_{j} \mathrm{~s}$ is equal to this fixed value.

To formulate this mathematically, we re-index the set $\left\{\left(\alpha_{j}, \beta_{j}\right) \square j=1, \ldots, M\right\}$ as follows:

Denote by $\left\{\alpha_{(k)} \square k=1, \ldots, K\right\}$ all different $\alpha_{j}$ values for $j=1, \ldots, M$. Denote by

$\left\{\beta_{(k, l)} \square l=1, \ldots, L_{k}\right\}$ all values $\beta_{j}$ corresponding to a fixed $\alpha_{(k)}$-value. Then we assume, for all $k=1, \ldots, K$ :

$$
\alpha_{k}=\frac{1}{L_{k}} \sum_{l=1}^{L_{k}} \beta_{(k, l)}
$$

Note that

$$
\sum_{k=1}^{K} L_{k}=M
$$

by definition. In other words

$$
\left\{\left(\alpha_{j}, \beta_{j}\right) \square j=1, \ldots, M\right\}=\left\{\left(\alpha_{(k)}, \beta_{(k, l)}\right) \square l=1, \ldots, L_{k} ; k=1, \ldots, K\right\}
$$

This assumption is used in this paper as an "axiom" but it is intuitively clear that, if we have many data points (i.e. if $M$ is high) that (9) can be assumed, based on Theorem 1 and the defining equations (7) and (8).

In the sequel we will also use the next simple Lemma.

\section{Lemma 1:}

Denote by $\bar{\alpha}$ the average of the values $\left\{\alpha_{j} \square j=1, \ldots, M\right\}$ and by $\bar{\beta}$ the average of the values $\left\{\beta_{j} \square j=1, \ldots, M\right\}$. Then

$$
\bar{\alpha}=\bar{\beta}
$$

\section{Proof:}

By definition,

$$
\begin{aligned}
\bar{\alpha}-\bar{\beta} & =\frac{1}{M} \sum_{j=1}^{M} \alpha_{j}-\frac{1}{M} \sum_{j=1}^{M} \beta_{j} \\
& =\frac{1}{M} \sum_{j=1}^{M}\left(\alpha_{j}-\beta_{j}\right)
\end{aligned}
$$




$$
=\frac{1}{M} \sum_{k=1}^{K} \sum_{l=1}^{L_{k}}\left(\alpha_{(k)}-\beta_{(k, l)}\right)
$$

by definition of $\alpha_{(k)}$ and $\beta_{(k, l)}$. But this last expression equals

$$
\bar{\alpha}-\bar{\beta}=\frac{1}{M} \sum_{k=1}^{K}\left(L_{k} \alpha_{(k)}-\sum_{l=1}^{L_{k}} \beta_{(k, l)}\right)=0
$$

by (9). Hence $\bar{\alpha}=\bar{\beta}$ is proved.

Based on this assumption we will be able to explain the three types of $A o R$ versus $R o A$ graphs in Larivière and Gingras (2011).

\section{$\underline{\text { RoA versus AoR }}$}

In Larivière and Gingras (2011) one studies $R o A$ versus $A o R$ in the interpretation of (6) and (7) ("crown indicator" versus "new crown indicator") in four cases: individuals (A), departments (B), institutions (C) and countries (D) for Canadian papers published in the period 2000-2008, based on Web of Science (WoS) data (more details can be found in Larivière and Gingras (2011)). Fig.1 (also Fig.1 in Larivière and Gingras (2011)) shows the relationship between $R O A$ in function (or versus) $A o R$. 

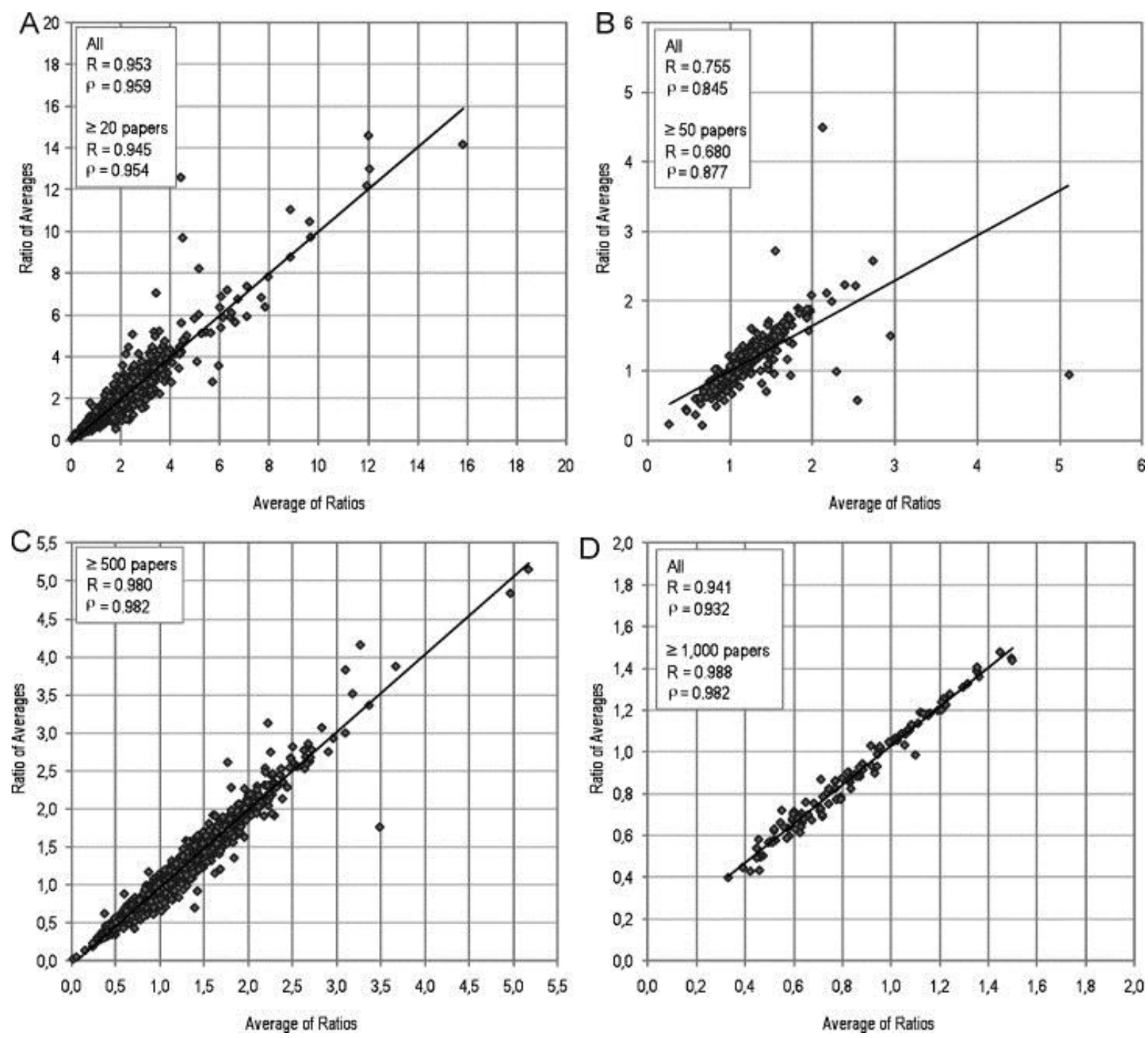

Fig. 1. Relation between RoA and AoR in 4 cases in Larivière and Gingras (2011).

It is intuitively clear that in all cases, the regression line of the cloud of points

$$
\left(\alpha_{j}, \beta_{j}\right)=\left(A o R_{j}, \operatorname{RoA}_{j}\right)
$$

$j=1, \ldots, M$ is the first bissectrix (even in case B the majority of the points follow the first bissectrix). This will be proved in the next theorem.

\section{Theorem 2:}

Denote by

$$
R=a+b A
$$

the regression line of the cloud of points $\left(\alpha_{j}, \beta_{j}\right)=\left(A o R_{j}, \operatorname{RoA}_{j}\right)$ for $j=1, \ldots, M$. Then $a=0$ and $b=1$, hence the first bissectrix $R=A$. 


\section{Proof}

According to classical statistics (see e.g. Egghe and Rousseau (2001), p.56), we have the following formulae for $a$ and $b$ :

$$
\begin{gathered}
b=\frac{\frac{1}{M} \sum_{j=1}^{M} \alpha_{j} \beta_{j}-\bar{\alpha} \square \bar{\beta}}{\frac{1}{M} \sum_{j=1}^{M} \alpha_{j}^{2}-\bar{\alpha}^{2}} \\
a=\bar{\beta}-b \bar{\alpha}
\end{gathered}
$$

So, to prove that $b=1$ we need to show that

$$
\frac{1}{M} \sum_{j=1}^{M} \alpha_{j} \beta_{j}-\bar{\alpha} \square \bar{\beta}=\frac{1}{M} \sum_{i=1}^{M} \alpha_{i}^{2}-\bar{\alpha}^{2}
$$

By Lemma 1 this boils down to showing that

$$
\sum_{j=1}^{M} \alpha_{j}\left(\beta_{j}-\alpha_{j}\right)=0
$$

But

$$
\sum_{j=1}^{M} \alpha_{j}\left(\beta_{j}-\alpha_{j}\right)=\sum_{k=1}^{K} \sum_{l=1}^{L_{k}} \alpha_{(k)}\left(\beta_{(k, l)}-\alpha_{(k)}\right)
$$

by the notation in the previous section. The last expression equals

$$
\sum_{k=1}^{K} \alpha_{(k)}\left(\sum_{l=1}^{L_{k}} \beta_{(k, l)}-\alpha_{(k)}\right)=\sum_{k=1}^{K} \alpha_{(k)}\left(\sum_{l=1}^{L_{k}} \beta_{(k, l)}-L_{k} \alpha_{(k)}\right)=0
$$

by (9). This proves that $b=1$.

But then (15), Lemma 1 and the fact that $b=1$ prove that $a=0$. Hence the regression line of the cloud of points $\left(\alpha_{j}, \beta_{j}\right)=\left(A o R_{j}, R o A_{j}\right)$ is the first bissectrix.

Note that assumption (9) already shows that, for every fixed $k=1, \ldots, K$, the averages of the values $\left\{\beta_{(k, l)} \square l=1, \ldots, L_{k}\right\}$ are equal to $\alpha_{(k)}$ and hence are on the first bissectrix, as a function of $\alpha_{(k)}$. But this does not yield a proof of Theorem 2 .

\section{$\underline{\text { Note }}$}

In Waltman et al. (2011b) similar graphs as in Fig. 1 are produced, yielding more practical evidence for validity of Theorem 2 . 
In return, the fact that the Assumption (9) is capable of proving Theorem 2, which is verified in practice (Fig.1), is a justification for the approximate validity of the Assumption.

\section{$($ AoR - RoA $) / A o R$ versus the number of papers}

If we look at Fig.2 (is also Fig.2 in Larivière and Gingras (2011)), we clearly see a cloud of points

$$
\left(N_{j}, \frac{A o R_{j}-R o A_{j}}{A o R_{j}}\right)=\left(N_{j}, \frac{\alpha_{j}-\beta_{j}}{\alpha_{j}}\right)
$$

$j=1, \ldots, M$, that is, more or less, symmetrically distributed around the $\mathrm{x}$-axis and whose thickness decreases (with increasing $N_{j}$ ) in a non-linear way). The first property can be understood if we calculate the average of the values

$$
\Delta_{j}=\frac{\alpha_{j}-\beta_{j}}{\alpha_{j}}
$$

for $j=1, \ldots, M$.

\section{Propostion 1:}

$$
\bar{\Delta}=\frac{1}{M} \sum_{j=1}^{M} \Delta_{j}=0
$$

\section{Proof:}

$$
\begin{aligned}
\frac{1}{M} \sum_{j=1}^{M} \Delta_{j} & =\frac{1}{M} \sum_{j=1}^{M} \frac{\alpha_{j}-\beta_{j}}{\alpha_{j}} \\
& =\frac{1}{M} \sum_{k=1}^{K} \sum_{l=1}^{L_{k}} \frac{\alpha_{(k)}-\beta_{(k, l)}}{\alpha_{(k)}} \\
& =\frac{1}{M} \sum_{k=1}^{K} \frac{\sum_{l=1}^{L_{k}}\left(\alpha_{(k)}-\beta_{(k, l)}\right)}{\alpha_{(k)}} \\
& =\frac{1}{M} \sum_{k=1}^{K} \frac{L_{k} \alpha_{(k)}-\sum_{l=1}^{L_{k}} \beta_{(k, l)}}{\alpha_{(k)}}=0
\end{aligned}
$$

by (9). 
The second property (decreasing thickness of the cloud of points, for increasing $N_{j}$ ) is a consequence of several results in probability theory and statistics. The main result that we use is the fact that a sample mean $\bar{x}$ (of size $N$ ) has a variance $V(\bar{x})$ equal to

$$
V(\bar{x})=\frac{\sigma^{2}}{N}
$$

, where $\sigma^{2}$ is the population variance (see e.g. Blom (1989), p.196). We will also use that the average of the $\bar{x}$ equals

$$
E(\bar{x})=\mu
$$

, the population mean (Blom (1989), p.196). We will also need the following results in Blom (1989), p.124, 125. For any random variable $X$ we have

$$
\begin{gathered}
E\left(\frac{1}{X}\right) \approx \frac{1}{E(X)} \\
V\left(\frac{1}{X}\right) \approx V(X)[E(X)]^{4}
\end{gathered}
$$

, where $\approx$ is Gauss's approximation. The product $X Y$ of two independent random variables satisfies the following relations:

$$
V(X Y) \approx V(X)[E(Y)]^{2}+V(Y)[E(X)]^{2}
$$

and

$$
E(X Y)=E(X) E(Y)
$$

We will use these relations since independence between the $\mathrm{x}$ - and $\mathrm{y}$-values can be supposed. We then have Theorem 3.

\section{Theorem 3:}

Denote by $\Delta$ one of the values $\frac{\alpha_{j}-\beta_{j}}{\alpha_{j}}$. Then

$$
V(\Delta) \square \frac{1}{N}
$$

, where $\sqcup$ means $\approx \frac{a}{N}$ where $a$ is a constant. 


\section{Proof:}

The above formulae (19) - (24) will be repeatedly used.

$$
\begin{gathered}
\Delta=\frac{\frac{1}{N} \sum_{i=1}^{N} \frac{y_{i}}{x_{i}}-\frac{\sum_{i=1}^{N} y_{i}}{\sum_{i=1}^{N} x_{i}}}{\frac{1}{N} \sum_{i=1}^{N} \frac{y_{i}}{x_{i}}} \\
\Delta=1-N \frac{\sum_{i=1}^{N} y_{i}}{\sum_{i=1}^{N} x_{i} \sum_{i=1}^{N} \frac{y_{i}}{x_{i}}} \\
\Delta=1-\frac{\bar{y}}{\overline{-\left(\frac{y}{x}\right)}}
\end{gathered}
$$

where $\overline{\left(\frac{y}{x}\right)}$ denotes

$$
\overline{\left(\frac{y}{x}\right)}=\frac{1}{N} \sum_{i=1}^{N} \frac{y_{i}}{x_{i}}
$$

, the average of the $\frac{y}{x}$-values. Hence,

$$
\begin{aligned}
& V(\Delta)=V\left(\frac{\bar{y}}{\bar{x}\left(\frac{y}{x}\right)}\right) \\
& \approx V(\bar{y})\left[E\left(\frac{1}{\bar{x}\left(\frac{y}{x}\right)}\right)\right]^{2}+V\left(\frac{1}{\bar{x}\left(\frac{y}{x}\right)}\right)[E(\bar{y})]^{2} \\
& \approx V(\bar{y})\left[E\left(\frac{1}{\bar{x} \overline{\left(\frac{y}{x}\right)}}\right)\right]^{2}+V\left(\bar{x} \overline{\left(\frac{y}{x}\right)}\right)\left[E\left(\overline{x\left(\frac{y}{x}\right)}\right)\right]^{4}[E(\bar{y})]^{2}
\end{aligned}
$$




$$
\begin{aligned}
& \approx V(\bar{y})\left[E\left(\frac{1}{\bar{x}\left(\frac{y}{x}\right)}\right)\right]^{2} \\
& +\left[V(\bar{x})\left[E\left(\overline{\left(\frac{y}{x}\right)}\right)\right]^{2}+V\left(\overline{\left(\frac{y}{x}\right)}\right)[E(\bar{x})]^{2}\right]\left[E\left(\bar{x} \overline{\left(\frac{y}{x}\right)}\right)\right]^{4}[E(\bar{y})]^{2} \\
& \approx \frac{\sigma_{y}^{2}}{N} \frac{1}{\mu_{y}^{2}}+\left[\frac{\sigma_{x}^{2}}{N} \frac{\mu_{y}^{2}}{\mu_{x}^{2}}+\frac{\sigma_{y / x}^{2}}{N} \mu_{x}^{2}\right] \mu_{y}^{6}
\end{aligned}
$$

But

$$
\begin{aligned}
\sigma_{y / x}^{2} & =V\left(\frac{y}{x}\right) \approx V(y)\left[E\left(\frac{1}{x}\right)\right]^{2}+V\left(\frac{1}{x}\right)[E(y)]^{2} \\
& \approx \sigma_{y}^{2} \frac{1}{\mu_{x}^{2}}+V(x)[E(x)]^{4} \mu_{y}^{2}
\end{aligned}
$$

So

$$
\sigma_{y / x}^{2} \approx \sigma_{y}^{2} \frac{1}{\mu_{x}^{2}}+\sigma_{x}^{2} \mu_{x}^{4} \mu_{y}^{2}
$$

and hence

$$
V(\Delta) \approx \frac{\sigma_{y}^{2}}{N} \frac{1}{\mu_{y}^{2}}+\left[\frac{\sigma_{x}^{2}}{N} \frac{\mu_{y}^{2}}{\mu_{x}^{2}}+\frac{1}{N}\left(\frac{\sigma_{y}^{2}}{\mu_{x}^{2}}+\sigma_{x}^{2} \mu_{x}^{4} \mu_{y}^{2}\right) \mu_{x}^{2}\right] \mu_{y}^{6}
$$

Since all $\sigma_{x}^{2}, \sigma_{y}^{2}, \mu_{x}, \mu_{y}$ are population variances and means, they are independent of $N$. Hence

$$
v(\Delta) \square \frac{1}{N}
$$

So, the standard deviation $(\sigma(\Delta)=\sqrt{V(\Delta)})$ is proportional with $\frac{1}{\sqrt{N}}$ and this determines the shape of the cloud of points in Fig.2: a decreasing thickness (in function of $\mathrm{N}$ ) proportional to $\frac{1}{\sqrt{N}}$. Of course, $\lim _{N \rightarrow \infty} \frac{1}{\sqrt{N}}=0$ showing that the decreasing thickness goes to 0 for $N$ going to $\infty$. 

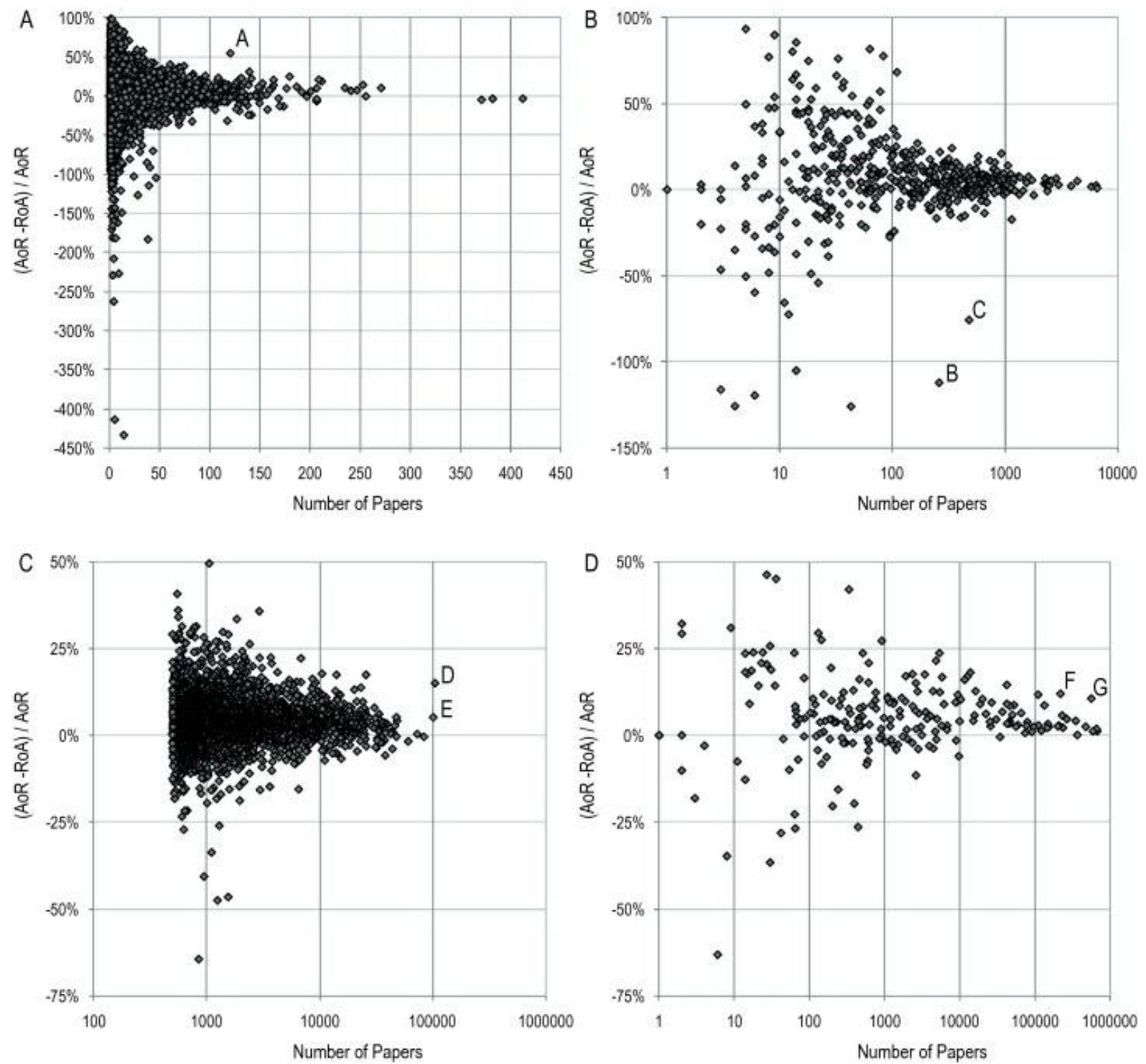

Fig. 2. Relationship between (AoR-RoA)/AoR and the number of papers in 4 cases in Larivière and Gingras (2011).

\section{$\underline{(\operatorname{AoR}-\operatorname{RoA}) / A o R}$ versus RoA}

If we look at Fig.3 (is also Fig.3 in Larivière and Gingras (2011)); we see a cloud of points

$$
\left(R o A_{j}, \frac{A o R_{j}-R o A_{j}}{A o R_{j}}\right)=\left(\beta_{j}, \frac{\alpha_{j}-\beta_{j}}{\alpha_{j}}\right)
$$

$j=1, \ldots, M$, that has a decreasing regression line. This will be proved in the next Theorem. 

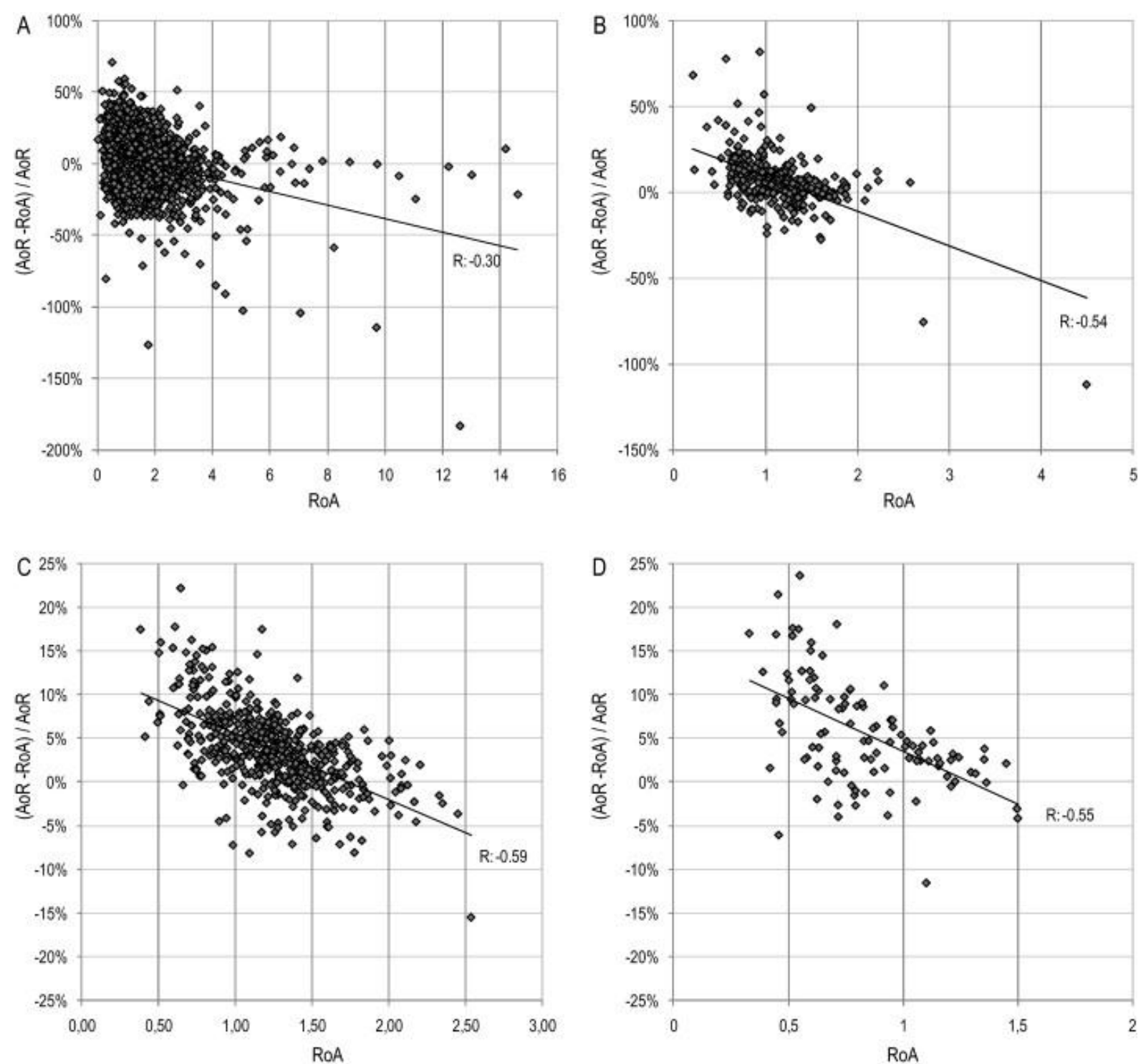

Fig. 3. Relation between (AoR-RoA)/AoR and RoA in 4 cases in Larivière and Gingras (2011).

\section{Theorem 4:}

The slope of the regression line of the cloud of points (30) is negative.

\section{Proof:}

The slope $b$ of the regression line of the cloud of points (30) is given by (see e.g. Egghe and Rousseau (2001))

$$
b=\frac{\frac{1}{M} \sum_{j=1}^{M} \beta_{j}\left(\frac{\alpha_{j}-\beta_{j}}{\alpha_{j}}\right)-\bar{\beta} \overline{\left(\frac{\alpha_{j}-\beta_{j}}{\alpha_{j}}\right)}}{\frac{1}{M} \sum_{j=1}^{M} \beta_{j}^{2}-\bar{\beta}^{2}}
$$

where 


$$
\overline{\left(\frac{\alpha_{j}-\beta_{j}}{\alpha_{j}}\right)}
$$

denotes the average of the values $\frac{\alpha_{j}-\beta_{j}}{\alpha_{j}}, j=1, \ldots, M$. Since the denominator is positive (being the variance of the $\beta_{j}$-values) we have to show that the numerator is negative, hence that

$$
\sum_{j=1}^{M} \beta_{j}\left(\frac{\alpha_{j}-\beta_{j}}{\alpha_{j}}\right)<\frac{1}{M}\left(\sum_{j=1}^{M} \beta_{j}\right)\left(\sum_{j=1}^{M} \frac{\alpha_{j}-\beta_{j}}{\alpha_{j}}\right)
$$

or

$$
\begin{aligned}
\sum_{j=1}^{M} \beta_{j}-\sum_{j=1}^{M} \frac{\beta_{j}^{2}}{\alpha_{j}} & <\frac{1}{M}\left(\sum_{j=1}^{M} \beta_{j}\right)\left(M-\sum_{j=1}^{M} \frac{\beta_{j}}{\alpha_{j}}\right) \\
& =\sum_{j=1}^{M} \beta_{j}-\frac{1}{M}\left(\sum_{j=1}^{M} \beta_{j}\right)\left(\sum_{j=1}^{M} \frac{\beta_{j}}{\alpha_{j}}\right)
\end{aligned}
$$

So we have to prove that

$$
\sum_{j=1}^{M} \frac{\beta_{j}^{2}}{\alpha_{j}}>\frac{1}{M}\left(\sum_{j=1}^{M} \beta_{j}\right)\left(\sum_{j=1}^{M} \frac{\beta_{j}}{\alpha_{j}}\right)
$$

Putting (33) in the notation of the Assumption, (33) becomes

$$
\sum_{k=1}^{K} \sum_{l=1}^{L_{k}} \frac{\beta_{(k, l)}^{2}}{\alpha_{(k)}}>\frac{1}{M}\left(\sum_{k=1}^{K} \sum_{l=1}^{L_{k}} \beta_{(k, l)}\right)\left(\sum_{k=1}^{K} \sum_{l=1}^{L_{k}} \frac{\beta_{(k, l)}}{\alpha_{(k)}}\right)
$$

But, using (9) twice, (34) is equivalent with

$$
\sum_{k=1}^{K} \sum_{l=1}^{L_{k}} \frac{\beta_{(k, l)}^{2}}{\alpha_{(k)}}>\frac{1}{M}\left(\sum_{k=1}^{K} L_{k} \alpha_{(k)}\right)\left(\sum_{k=1}^{K} L_{k}\right)
$$

Now, using (10), (35) is equivalent with

$$
\sum_{k=1}^{K} \sum_{l=1}^{L_{k}} \frac{\beta_{(k, l)}^{2}}{\alpha_{(k)}}>\sum_{k=1}^{K} L_{k} \alpha_{(k)}
$$

For (36) it suffices to prove that, for every $k=1, \ldots, M$

$$
\sum_{l=1}^{L_{k}} \frac{\beta_{(k, l)}^{2}}{\alpha_{(k)}}>L_{k} \alpha_{(k)}
$$

or

$$
\sum_{l=1}^{L_{k}} \beta_{(k, l)}^{2}>L_{k} \alpha_{(k)}^{2}
$$


But, again using (9), we see that

$$
\sum_{l=1}^{L_{k}} \beta_{(k, l)}=L_{k} \alpha_{(k)}
$$

Hence

$$
L_{k}^{2} \alpha_{(k)}^{2}=\left(\sum_{l=1}^{L_{k}} \beta_{(k, l)}\right)^{2} \leq\left(\sum_{l=1}^{L_{k}} \beta_{(k, l)}^{2}\right) L_{k}
$$

by the inequality of Cauchy-Schwarz. Now (38) implies (37), supposing that not all $\beta_{(k, l)}$, $l=1, \ldots, L_{k}$ are equal, for every $k=1, \ldots, K$ (in which case the studied regression line is horizontal and all points are on the regression line - a trivial case which we exclude here). This ends the proof of this theorem.

\section{Conclusions and comments}

We proved that, using a simple assumption, the regression line of $R o A$ in function of $A o R$ is the first bissectrix. We also showed that the cloud of points $(A o R-R o A) / A o R$ in function of $N$ (the number of papers) is comprised between a multiple of $1 / \sqrt{N}$ and $-1 / \sqrt{N}$. Finally we showed that the slope of the regression line of $(A o R-R o A) / A o R$ in function of $R o A$ is negative.

In this way we could explain the three graphs that were published in Larivière and Gingras (2011) in an informetrically consistent way. We do not go into the "Dutch" discussion described in the introduction. We, simply, want to make a few remarks.

(i) As shown in Egghe and Rousseau (1996b), using the geometric average in the definition of $A o R$ and $R o A$ (instead of the arithmetic average), yields the same indicator. The proof is very short and is repeated here. Replacing in (1) the arithmetic average by the geometric one yields the indicator denoted $g(A o R)$ :

$$
g(A o R)=\left(\frac{y_{1}}{x_{1}} \frac{y_{2}}{x_{2}} \ldots \frac{y_{N}}{x_{N}}\right)^{\frac{1}{N}}
$$

Doing the same in formula (2), now denoted $g(\operatorname{RoA})$, we have (use formula (3)) 


$$
g(R o A)=\frac{\left(y_{1} y_{2} \ldots y_{N}\right)^{\frac{1}{N}}}{\left(x_{1} x_{2} \ldots x_{N}\right)^{\frac{1}{N}}}
$$

Hence, trivially

$$
g(A o R)=g(R o A)
$$

(ii) The fact that the regression line of $R o A$ in function of $A o R$ is the first bissectrix (as shown here) and the fact that the cloud of points is very close to this line (see Fig.1) shows that $R o A$ and $A o R$ are very similar valuewise (in most cases).

(iii) The fact that the cloud of points $(A o R-R o A) / A o R$ in function of $N$ (the number of papers) is centered around the $\mathrm{x}$-axis and that its thickness goes to zero for $N$ large shows again that $A o R$ and $R o A$ are very similar valuewise (in most cases).

(iv) In Egghe, Rousseau and Van Hooydonck (2000) we showed, however, that rankings based on $R o A$ can be very different from rankings based on $A o R$. 


\section{$\underline{\text { References }}$}

G. Blom (1989). Probability and Statistics. Theory and Applications. Springer-Verlag, New York, USA.

L. Egghe and R. Rousseau (1996a). Average and global impact of a set of journals. Scientometrics 36(1), 97-107.

L. Egghe and R. Rousseau (1996b). Averaging and globalizing quotients of informetric and scientometric data. Journal of Information Science 22(3), 165-170.

L. Egghe and R. Rousseau (2001). Elementary Statistics for Effective Library and Information Management. ASLIB imi Information Management, London, UK.

L. Egghe and R. Rousseau (2002/2003). A general framework for relative indicators. Cadre général pour les facteurs d'impact relatifs. The Canadian Journal of Information and Library Science / La Revue canadienne des science de l'information et de bibliothéconomie 27(1), 29-48.

L. Egghe, R. Rousseau and G. Van Hooydonk (2000). Methods for accrediting publications to authors or countries: consequences for evaluations studies. Journal of the American Society for Information Science 51(2), 145-157.

V. Larivière and Y. Gingras (2011). Averages of ratios vs ratios of averages: An empirical analysis of four levels of aggregation. Journal of Informetrics, to appear.

T. Opthof and L. Leydesdorff (2010). Caveats for the journal and field normalizations in the CWTS ("Leiden") evaluations of research performance. Journal of Informetrics 4(3), 423-430.

R. Rousseau and L. Leydesdorff (2011). Simple arithmetic versus intuitive understanding: The case of the impact factor. ISSI Newsletter

L. Waltman, N.J. van Eck, T.N. van Leeuwen, M.S. Visser and A.F.J. van Raan (2011a). Towards a new crown indicator: Some theoretical considerations. Journal of Informetrics 5(1), 37-47.

L Waltman, N.J. van Eck, T.N. van Leeuwen, M.S. Visser and A.F.J. van Raan (2011b). Towards a new crown indicator: An empirical analysis. Scientometrics 87, 467-481. 